\section{A first for monkey models}

\section{By Tracey Baas, Associate Editor}

The inability to produce transgenic nonhuman primates that pass transgenes to their progeny has been a major obstacle to studying inherited diseases and conditions determined by disease susceptibility genes. Japanese researchers have developed a potential solution: marmosets that pass on a transgene to their progeny. ${ }^{1}$

If the approach can be refined, biotech service providers may have to play catch-up, as there are only limited marmoset-related genomic data available and a dearth of marmoset-specific reagents such as antibodies, microarrays, cytokines and cDNA clones.

Rhesus macaques are the most commonly used monkey model, but transgenic production of these large primates has been difficult because of low transgene incorporation rates. ${ }^{2-4}$ Macaques

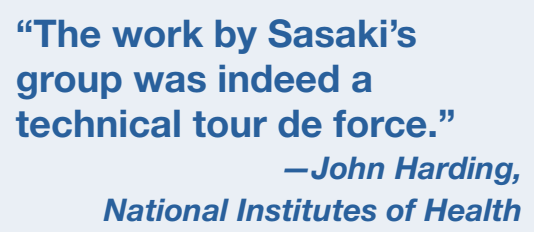

Research Center and in the Department of Human Genetics at the Emory University School of Medicine.

"The researchers have been able to advance the technology such that a gene of interest can be expressed with a $100 \%$ success rate if a pregnancy occurs in a nonhuman primate," said Marie Kosco-Vilbois and Luca Bolliger in joint comments to SciBX. Kosco-Vilbois is CSO and Bolliger is director of business development at NovImmune S.A.

Kosco-Vilbois and Bolliger did note that "there are mountains worth of refinement required to master the technology in this species," such as honing integration sites and generating knock-ins versus knockouts.

The good news, they said, is that the reproductive speed and size of marmosets could "facilitate the adoption of this technology in both contract research organizations as well as academic environments. However, in parallel, these advances catalyze the need of a broader ethical discussion within an ever changing modern society."

"The work by Sasakis group was indeed a technical tour de force," said John Harding, director of primate resources at the NIH's National Center for Research Resources.

He did say that many researchers "would prefer not to use viral vector gene delivery, which results in random transgene integration." There are approaches that could allow for site-specific transgene incorporation, such as using embryonic stem cells, he noted. "But rhesus embryonic stem cells have not yet been demonstrated to be also take 3 years to reach sexual maturity and only produce about 10 offspring in their lifetime.

In contrast, marmosets reach sexual maturity at $12-18$ months and are capable of producing $40-80$ offspring.

The Japanese team transformed marmoset embryos in vitro with a selfinactivating lentiviral vector that delivered a transgene encoding enhanced green fluorescent protein (EGFP). They used natural embryos flushed from mated female marmosets, $100 \%$ of which expressed EGFP. The viral vector was able to randomly insert the transgene into the genome of both somatic and germline cells.

Researchers then implanted 50 marmosets with 80 EGFP-expressing embryos, resulting in 4 infants that had the transgene incorporated in their somatic cells. Two of those infants also had the transgene incorporated in their germ cells. When these first-generation marmosets reached sexual maturity, they produced three EGFP-expressing second-generation marmosets.

That group of animals was the first-ever example of a transgenic nonhuman primate with germline transmission.

The research group was led by Erika Sasaki, head of the laboratory of applied developmental biology at the Central Institute for Experimental Animals, and included investigators at several other Japanese universities and institutions.

The reproductive advantage of marmosets and their ability "to pass a transgene to the next generation allows researchers to investigate the impact of inherited genetic disease in different generations," noted Anthony Chan, assistant professor at the Yerkes National Primate capable of germline transmission of transgenes. Alternatively, induced pluripotent stem cells could open up parallel approaches for working with rhesus, or marmoset, stem cells."

Embryonic stem cells are routinely used to produce transgenic mice.

\section{Where to start}

With the newfound ability to create transgenic marmosets with germline gene integration, the open question is what diseases to investigate first.

One idea is to use the model for diseases in which rodent models haven't yielded good therapies.

Jeffrey Chamberlain, an expert in muscular dystrophy (MD), thinks it would be worth trying the model in MD given that mice and dogs, the conventional animal models for Duchenne muscular dystrophy (DMD), have multiple limitations. Chamberlain is a professor in the Department of Neurology, Medicine and Biochemistry at the University of Washington.

Mice with mutant dystrophin genes tolerate many therapeutics that may not be safe in humans, thus giving little if any useful information for dosing studies. In particular, mice "do not develop much of an immune response against certain treatment regimens-some gene-therapy vectors, for example-that do induce immune responses in larger animals, such as dogs," Chamberlain told SciBX.

"The marmoset is so close to humans that they would likely be much better at modeling immune reactions against an intervention and may well be a much better predictor of how an intervention would fare in the clinic," he added. 
Dog models exhibit variable weakness, which makes it unclear whether a therapeutic is increasing strength or whether a given dog is simply stronger than most. "Ideally, a marmoset model would display uniform and reproducible weakness, muscle wasting and time course of muscle degeneration," Chamberlain said.

Chan said "one good example that the transgenic marmosets could be the most useful for is investigating inherited diseases such as Huntington's disease," in which the size of the polyglutamine tract in the mutant huntingtin gene changes from generation to generation and correlates with disease severity and clinical outcome.

Because the marmosets can pass genes onto progeny, it might be possible to track what length of gene the new marmosets received and correlate this with disease severity.

Chan led the 2008 development of the first transgenic nonhuman primate model of Huntington's at Yerkes by producing mutant human huntingtin (HTT)-expressing rhesus macaques. Those animals are expected to produce offspring when they reach puberty at three to four years of age.

He did have one caveat: marmosets have very small brains, thus making it hard to run cognitive behavioral studies.

Nicole Déglon, assistant director of the Molecular Imaging Research Center at the French Atomic Energy Commission's Department of Medical Research, raised similar limitations. Although marmosets could be used for behavioral cognitive testing, she said, their performance "is inferior to that of larger primate species, in particular macaques, baboons and, of course, chimpanzees."

Even tests of fine motor skills, which are "readily performed by larger species," would need to be modified for marmosets, she said.

Déglon told SciBX the small brains also make marmosets suboptimal for both imaging and dose-ranging studies.

"Given that the marmoset brain is not much larger than that of a rat, this means that only large structures-striatum, cortex, hippocampus-can be assessed with the current limitations of positron emission tomography spatial resolution," she said. As a result, Déglon said she has stopped using marmosets to study diseases with cognitive degeneration.

Déglon also said marmosets might not provide an advantage in dosefinding studies. "There is not much advantage to using the marmosets rather than rodent models for scaling up intracerebral interventions or delivery of therapeutic molecules," she argued. "These would be better assessed in a large nonhuman primate model, such as for gene transfer where $40-80 \mu \mathrm{l}$ are needed for a large primate as compared to $300-500 \mu \mathrm{l}$ for a patient, but only $2 \mu \mathrm{l}$ for a mouse or rat or marmoset."

Sasaki countered that marmosets may be suitable for studying the effects of therapeutics on motor function in diseases such as Parkinson's, 5 and added that one of her team members is developing tests of cognitive function in marmosets.

Using the approach described in the Nature paper, Sasaki's group is working on establishing marmoset models of disease. The team is also working on modifying the technique to enable the introduction of transgenes that are larger than the current size limit of $8.5 \mathrm{kBs}$.

Sasaki said she is most interested in establishing models of familial Parkinson's disease (PD) and recessive juvenile amyotrophic lateral sclerosis (ALS). She said her group, which is participating in a five-year project called The Strategic Research Program for Brain Sciences, "could produce a Parkinson's disease model in the near future."

The program is aimed at understanding brain function and developing animal models for Alzheimer's disease (AD), PD and depression.

A patent application covering the work has been filed by the Central Institute for Experimental Animals and is available for licensing.

Baas, T. SciBX 2(24); doi:10.1038/scibx.2009.964

Published online June 18, 2009

\section{REFERENCES}

1. Sasaki, E. et al. Nature; published online May 28, 2009;

doi:10.1038/nature08090

Contact: Erika Sasaki, Central Institute for Experimental Animals,

Kawasaki, Japan

e-mail: esasaki@ciea.or.jp

Contact: Hideyuki Okano, Keio University School of Medicine, Tokyo,

Japan

e-mail: hidokano@sc.itc.keio.ac.jp

2. Chan, A.W. et al. Science 291, 309-312 (2001)

3. Wolfgang, M.J. et al. Proc. Natl. Acad. Sci. USA 98,10728-10732 (2001)

4. Yang, S.H. et al. Nature 453, 921-924 (2008)

5. Ando, K. et al. Psychopharmacology 195, 509-516 (2008)

\section{COMPANIES AND INSTITUTIONS MENTIONED}

Central Institute for Experimental Animals, Kawasaki, Japan Emory University School of Medicine, Atlanta, Ga.

French Atomic Energy Commission, Fontenay-aux-Roses, France National Institutes of Health, Bethesda, Md.

Novlmmune S.A., Geneva, Switzerland

University of Washington, Seattle, Wash.

Yerkes National Primate Research Center, Atlanta, Ga. 